\title{
Performance Enhancement of Cooperative Cognitive Relay Network using Multi Antennas Multi Hopping Techniques
}

\author{
Nada Ebaid \\ Faculty of Electronic Engineering \\ El-Menoufia University \\ Egypt
}

\author{
Mona Shokair \\ Faculty of Electronic Engineering \\ El-Menoufia University \\ Egypt
}

\author{
Sami A. El Dolil \\ Faculty of Electronic Engineering \\ El-Menoufia University \\ Egypt
}

\begin{abstract}
In this paper, two systems will be introduced to improve the performance of Cooperative Cognitive Relay Network (CCRN). The first is Compress-and-Forward (CF) relay scheme based on joint source-channel coding for threeterminal classical relay network. Simulation results show that CF relay scheme gives remarkable performance gains over other cooperation strategies such as decode-and-forward and amplify-and-forward in this scenario where both source-relay and relay destination links have low signal-to-noise ratios. The second system is multi-hopping cooperative relaying with multi-antennas using decode and forward scheme. Using multi-hopping with multi-antennas techniques in CCRN gives a remarkable enhancement in the performance than using only one hop with single antenna system.
\end{abstract}

\section{Keywords}

Compress and forward relay, decode and forward relay, multiantennas, multi-hopping and cognitive radio network.

\section{INTRODUCTION}

Cognitive Radio Network (CRN) is a future promising technology that is used to solve the spectrum scarcity problem. In fact, this is due to that the allocated spectrum for wireless networks is characterized by a fixed assignment policy. The Federal Communication Commission (FCC) attributed the reason of spectrum scarcity to the heavy underutilization of the allocated spectrum. The allocated spectrum in CRN is used in a dynamic manner to be capable of sensing and adapting to the environments. There are two types of users: unlicensed user referred to as Secondary User (SU) which can access the unused spectrum of the licensed user that referred to as Primary User (PU) without disturbing the communication of the primary user. Wireless networks are exposed to different channel problems such as fading and path loss. To overcome these problems, a relay will be used. In information theory, a relay channel is probability model of the communication between a sender and a receiver aided by one or more intermediate relay nodes. There exist three main relaying schemes: Decode andForward, Compress-and-Forward, and Amplify-and-Forward. The first two schemes were first proposed by Cover and ElGamal in [1]. In the decode-and-Forward (DF) scheme, the relay decodes the source message in one block and transmits the re-encoded message in the following block. In the compress-and-Forward (CF) scheme, the relay quantizes the received signal in one block and transmits the encoded version of the quantized received signal in the following block. In amplify-and-Forward (AF) scheme, the relay sends an amplified version of the received signal in the last time- slot. Comparing with $\mathrm{DF}$ and $\mathrm{CF}, \mathrm{AF}$ requires much less delay as the relay node operates time-slot by time-slot. Also, AF requires much less computing power as no decoding or quantizing operation is performed at the relay side but $\mathrm{CF}$ and $\mathrm{DF}$ overcome $\mathrm{AF}$ in performance as discussed in [2]. $\mathrm{CF}$ gives good performance on the scenarios where neither DF nor AF has a good performance due to low signal-to-noise ratios (SNR) on the source-to-relay (SR) and RD channels [3]. The relay node can employ standard source coding, or the WynerZiv Coding (WZC) technique when compressing the signal. The CF protocol with WZC at the relay following the rate distortion theory with side information [4] could support a slightly higher achievable rate in theory, compared with standard source coding [5]. However, for the WZC technique in practice, how to efficiently take advantage of the statistical dependence between the relay and the destination, and how to realize the theoretical performance limit of the CF protocol, are still open problems. If there exist multiple independent transmitters, the performance of CF protocol with WZC will be impaired by a larger compression noise when employing side information. Since standard source coding is much simpler for the CF protocol and also performs well in practical scenarios, it will be used at the relay.

In this paper, compress-and-forward relay scheme will be proposed using joint source-channel coding techniques. The relay performs scalar quantization (SQ) of its observation in combination with convolution coding and BPSK modulation. Code construction is based on simple code concatenation for joint source-channel coding at the relay and Viterbi decoding at the destination. There are some techniques to improve the performance of CCRN. One of these techniques is multihopping [6]. Single and multi-antennas multi-hop (multirelay) technique are studied with amplify and forward protocol in [7] but in this paper decode and forward protocol will be applied which are not clarified until now. This paper presents how infrastructure relays with/without multiple antennas can be used for providing diversity gains for a wireless communication and this will be applied on multi-hop system. The concept of multihop and multi-antennas diversity will be introduced where the benefits of spatial diversity are achieved from the concurrent reception of the signal that has been transmitted by multiple previous terminals and multiple antennas. The performance of multihop relay network will be investigated.

The rest of this paper will be organized as follows: Section 2 will present cooperative cognitive relay network. Section 3 will introduce compress and forward system model. In Section 4, multi-antennas multi-hopping system will be introduced. Simulation results will be made in section 5 . Finally, conclusions will be presented in Section 6. 


\section{COOPERATIVE COGNITIVE RELAY NETWORK (CCRN)}

Cooperative cognitive relay network has been recently proposed as a promising technology to improve the utilized efficiency of radio spectrum. It allows secondary user network to coexist with primary user networks through spectrum sharing, provided that the secondary spectrum access will not affect the PU's performance. PU in CCRN selects some secondary users to relay the traffic of its transmitter cooperatively toward the intended destination. The primary link gives out a portion of the channel access time to the selected SUs. Primary user decide to lease the spectrum for a fraction of time to the SUs in exchange for their cooperation in relaying the primary data [8].Providing service for PUs, improving the primary transmission performance, so that the PUs are willing to yield transmission opportunities for SUs as a reward [9]. This paper for simplify will refer to primary user transmitter as a source and primary user receiver as a destination and secondary users as hops or relays.

\section{COMPRESS AND FORWARD SYSTEM MODEL}

Relay channels (source-relay, source-destination, and relaydestination) are considered to be half-duplex independent Rayleigh fading channels with Additive White Gaussian Noise (AWGN). The channels are considered to be slow fading so that the coefficients are constant over the time duration of one symbol, that is, over one time slot. The channel coefficients and AWGN signals are all mutually independent. Throughout this paper transmissions over the different channels are assumed to be interference-free (the channels are orthogonal).
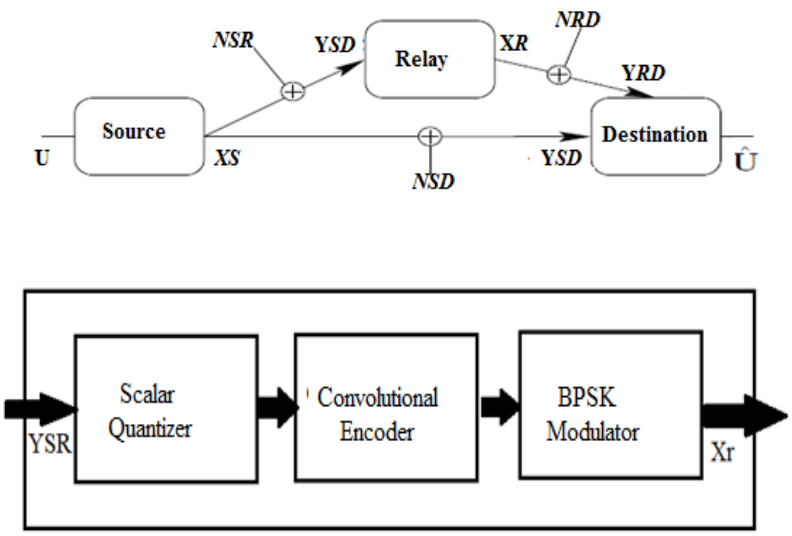

Fig.1: System model of a classical relay network with compress and forward relay scheme.

As illustrated in Figure 1, communication is divided into two phases. In the first one, the source transmits its signal Xs and both relay and destination observe noisy versions of this transmitted signal, YSR and YSD, respectively. After this phase is completed, the relay compresses its observation using Scalar Quantizer (SQ) which is a mapping of an input value into a finite number of output values. Scalar quantization is the most common type of quantization. It typically denoted as, the process of using a quantization function to map a scalar (one-dimensional) input value to a scalar output value. Scalar quantization can be as simple and intuitive as rounding high precision numbers to the nearest integer or to the nearest multiple of some other unit of precision. The next step is processing the output signal by using a combination of convolutional coding and Binary Phase Shift Keying (BPSK) modulator. Using source coding tools in compress and forward relay scheme leads to eliminate this superfluous information and then perform channel coding to combat errors on the RD link by introducing controlled redundancy [10]. Then the signal $X r$ will be sent during the second phase of communication. The destination observes a noisy version YRD of the relay transmission and makes BPSK demodulation. A Viterbi decoder uses the Viterbi algorithm for decoding the demodulated signal to recover the quantized signal W. Finally Maximum Ratio Combining (MRC) is implemented to combine the two received signals from the source (YSD) and relay (YRD), and makes a decision on the transmitted signal.

\section{MULTI-ANTENNAS MULTI- HOPPING SYSTEM MODEL}

In CCRN using secondary users as cooperative relays with multi-antennas to relay the signal of $\mathrm{Pu}$ will achieve the advantages of spatial diversity which is a powerful communication receiver technique that provides wireless link improvement at a relatively low cost. Diversity techniques are used in wireless communications systems to primarily improve performance over a fading radio channel. In such a system, the receiver is provided with multiple copies of the same information signal which is transmitted over two or more real or virtual communication channels. The source transmits symbols to the destination with the help of the $K$ relays, using a time orthogonal DF protocol over $K+1$ time slots (one for the source and one for each of the relays). All channels are Rayleigh fading channels with additive white Gaussian noise (AWGN) .As illustrated in Figure 2 using two antennas in one hop (one relay) system means that the signal transmitted from the source will be broadcasted over two different channels. The received signals at the relay at the first time slot can be expressed by,

$$
y_{s, i_{n}}=\sqrt{P_{s}} h_{s, i_{n}} x+n_{s, i_{n}}
$$

(1) where $x$ is the zero-mean and unit-energy transmitted symbol, $P_{S}$ is the source power and $h_{S, i n}$ is the channel response between source and relay number i with antenna number $n . n_{s, i_{n}} \sim C N\left(0, \sigma_{s, i_{n}}^{2}\right)$ is the complex Gaussian noise samples between source and relay. The received signal at the destination at the same time slot is given by,

$y_{S D}=\sqrt{P_{S}} h_{S D} x+N_{S D}$

(2)

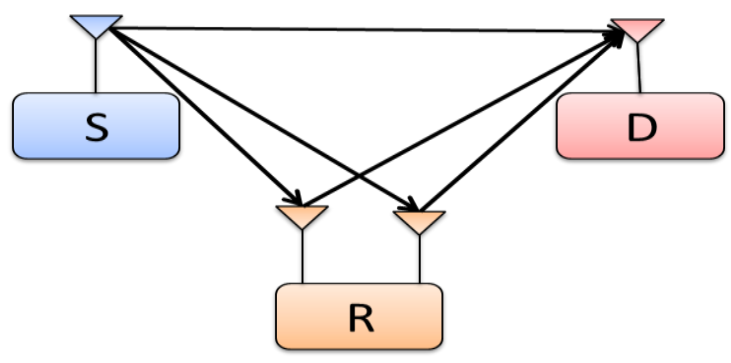

Fig. 2: One hop with two antennas system model.

At the second time slot relay will combine the two received signals using MRC. In MRC, suitable weight to each receiving signal will be selected depending on the value of the SNR of the receiving signal at the receiving node. The combined signal will be decoded and re-encoded. Relay send re-encoded signal to the destination using its two antennas on 
two different channels. Finally, received signals at the destination from the direct link and relay links through all time slots will be maximal ratio combined. By increasing the number of hops( relays) with two antennas at each relay to two hops, it gives the signal the chance to go through more paths than one hop with two antennas and two hops with one antenna. At the first time slot, the source transmits its signal to the destination and also to the two relays with two antennas through four different paths. In the second time slot, the received signals at each relay will be combined using MRC and the combined signal at each relay will be decoded and reencoded and forward to the destination and also the first relay will transmit its re-encoded signal to the second relay through different four paths. At the third time slot, the second relay will combine the four received signals and then the combined signal will be decoded, re-encoded and then broadcasted through the two antennas of the second relay to the destination. Finally at the destination, all the received signal through the three time slots will be maximal ratio combined, Viterbi decoded and BPSK demodulated. Then it makes a decision on the transmitted signal.
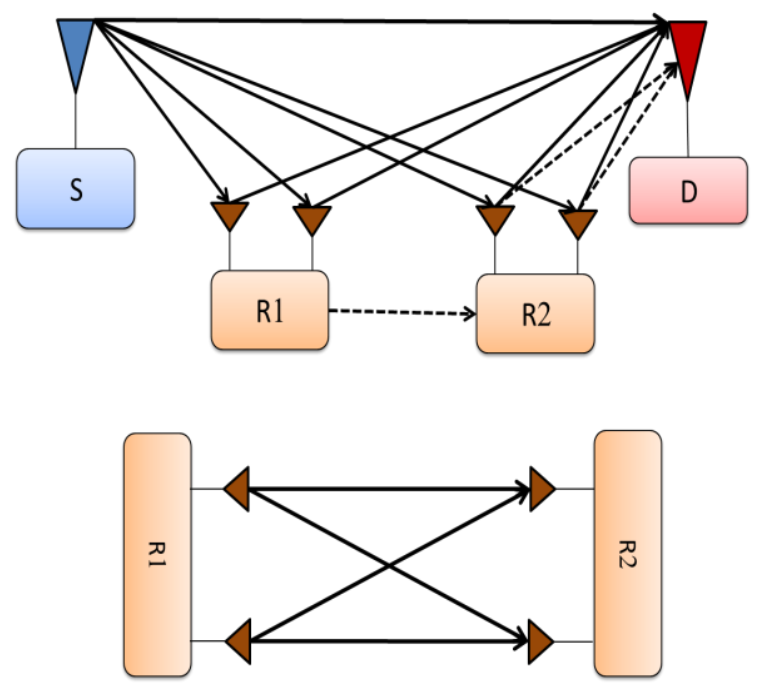

Fig. 3: Two hops with two antennas system model

\section{SIMULATION RESULTS}

The performance of compress and forward relay system and the multi-hopping multi antenna system will be evaluated with simulations in CCRN. Source messages consist of $10^{6}$ uniform bits protected with a recursive systematic convolutional code and modulated with BPSK. The compress and forward relay system presented here uses scalar quantizer with 2, 4, 6 and 8 levels and convolution codes with rate 1/2 and also uses BPSK modulation scheme. In this part, an approximate Bit Error Rate (BER) will be presented versus signal to noise ratio for the multi-hop system with $\mathrm{K}$ relays with a different number of antennas at the relay using (BPSK) scheme. All the results that will be taken after all signals from all channels are combined using MRC. Figure 4 shows the simulation of the direct link, AF and DF protocols. From this figure, it can be concluded that significant improvement in performance is observed by using DF protocol than using $\mathrm{AF}$ or the direct link.

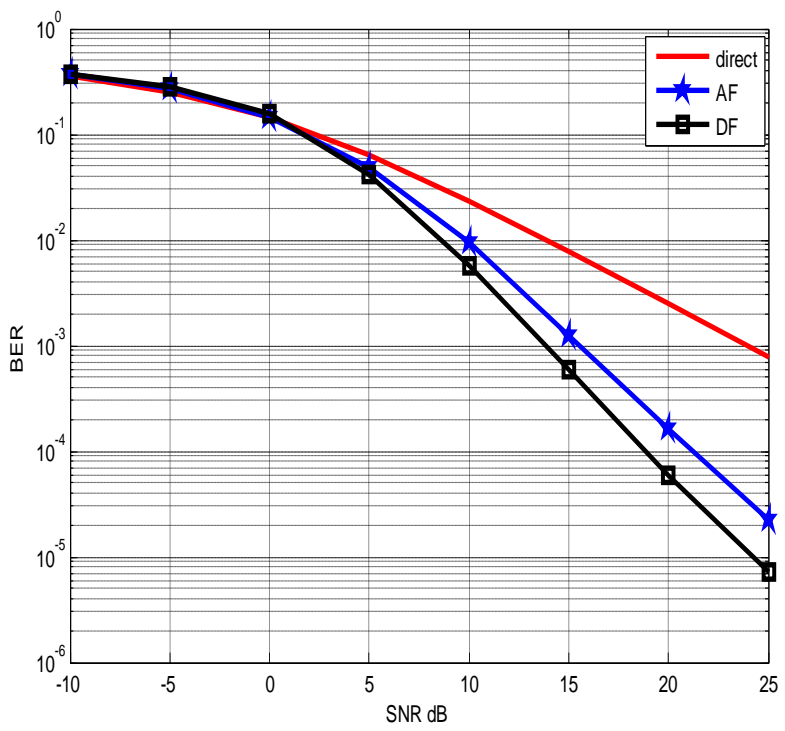

Fig. 4: Performances comparison for AF and DF with BPSK modulation scheme

In Figure 5, decode and forward one hop single and multiantenna simulation result will be presented. It is found that the performance of decode-and-forward relaying using two antennas at the relay in one hop system model is better than using one antenna at the relay in also one hop system model. For example at $\mathrm{SNR}=15 \mathrm{~dB}$ and BPSK modulation scheme using one hop with one antenna give BER equal to $6 * 10^{-4}$ but by increasing number of antennas to two antennas BER will reduce to $10^{-4}$.

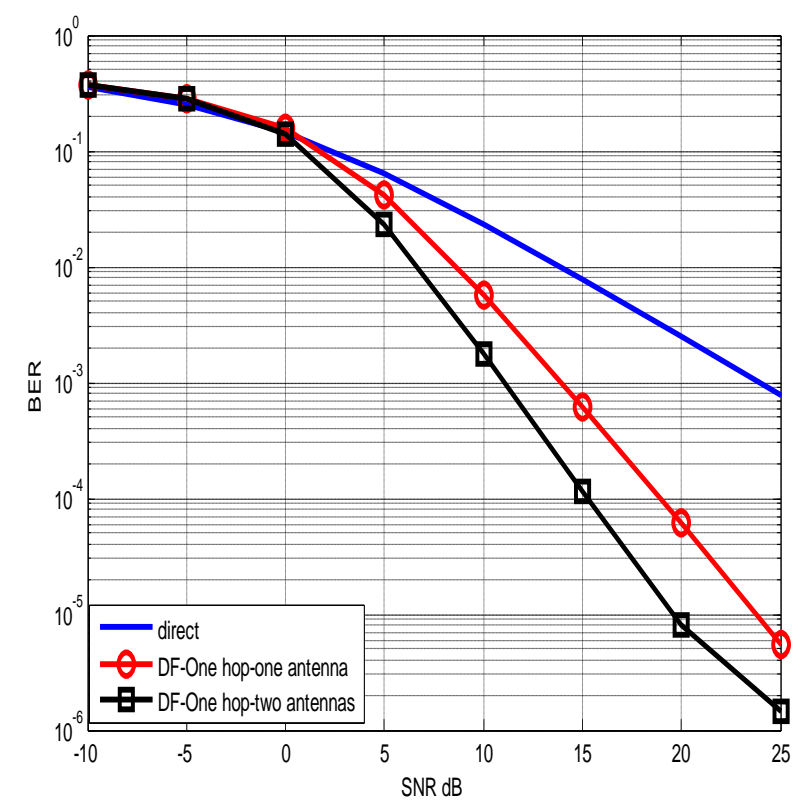

Fig. 5: BER performances for DF with BPSK scheme in one hop with one and two antennas

Figure 6 shows that increasing the number antennas from one antenna to two antennas in two hops techniques give a remarkable improvement on the performance of the system. For example at $\mathrm{SNR}=15 \mathrm{~dB}$ and BPSK modulation scheme using two hops with one antenna give BER equal to $2^{*} 10^{-4}$ but by increasing the number of antennas to two antennas 
BER will reduce to $10^{-6}$. These results prove that by increasing the number of hops and the number of antennas the performance of the system will give remarkable improvement in the performance of the system.

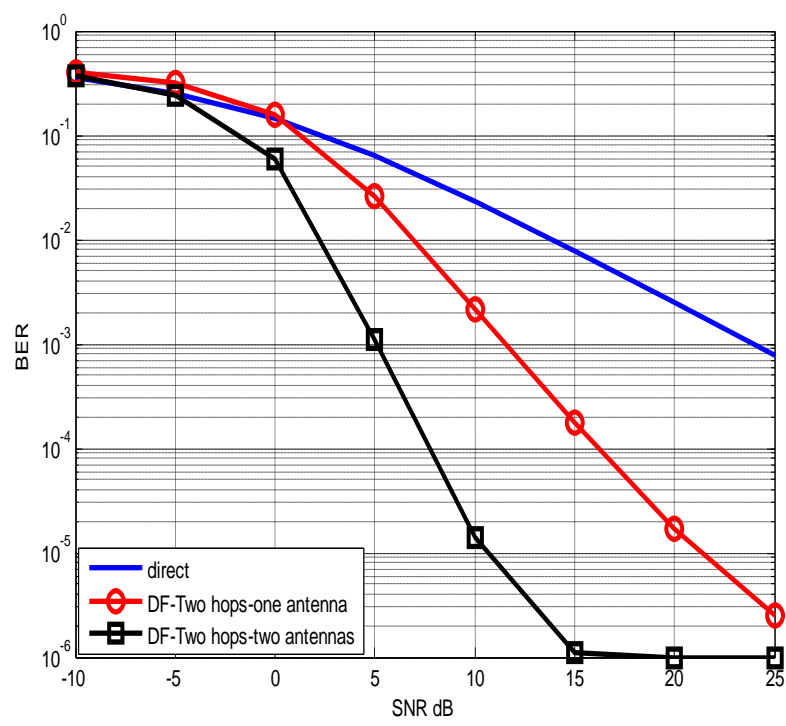

Fig. 6: BER performances for DF with BPSK scheme in two hops one and two antennas

It is clear from Figure 7 that using two hops with two antennas gives the best performance in the multi-hopping system.

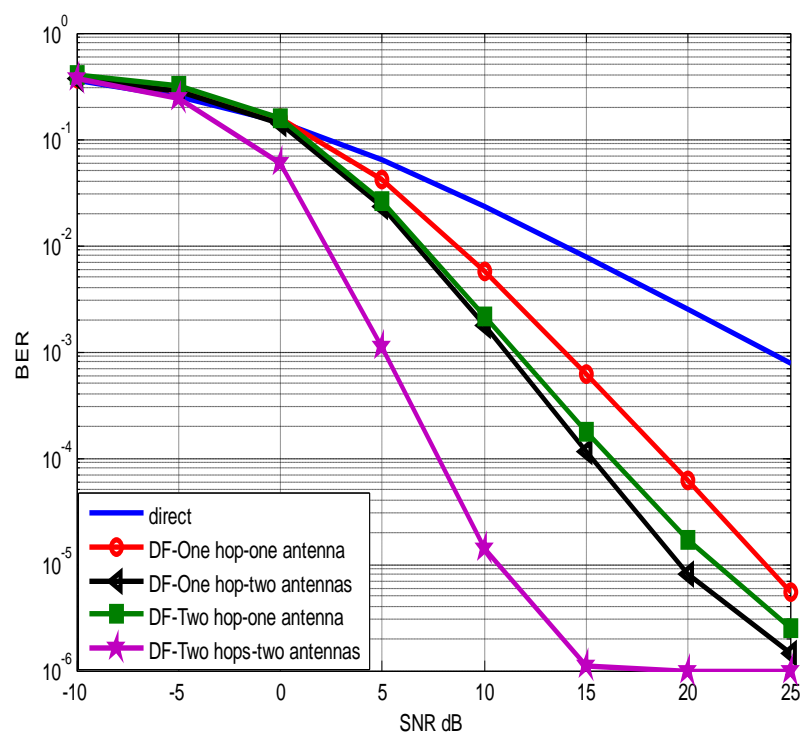

Fig.7: BER performances for DF with BPSK scheme multi-hopping system

Figure 8 shows the simulation of direct link, AF, DF and CF with different quantization rates. Using $\mathrm{CF}$ relay scheme is one of the solutions to minimize the disadvantages of $\mathrm{AF}$ and DF relay technique as mentioned earlier especially at low SNR. From this figure, it can be concluded that significant improvement in performance is observed by using $\mathrm{CF}$ technique than using $\mathrm{AF}, \mathrm{DF}$ and the direct link under BPSK modulation in terms of signal to noise ratio and bit error rate. For example at $\mathrm{SNR}=5 \mathrm{~dB}$, the BER approximately equal to $8 * 10^{-3}$ at $\mathrm{CF}, 4 * 10^{-2}$ at $\mathrm{DF}$ protocol, $5^{*} 10^{-2}$ at $\mathrm{AF}$ protocol and $6 * 10^{-2}$ at the direct link. The numerical results indicate that $\mathrm{CF}$ relay outperforms the direct, $\mathrm{AF}$ and $\mathrm{DF}$ relay technique. By increasing the quantization rate in $\mathrm{CF}$ relaying technique it can be observed that at the same value of SNR a remarkable decrease in BER will occur. At instant $\mathrm{SNR}=5 \mathrm{~dB}$ using $\mathrm{CF}$ with quantization rate equal to 4 bit per sample BER decreases to $6 * 10^{-3}$ and at quantization rate 6 bit per sample BER becomes $3 * 10^{-3}$. Increasing quantization rate to 8 bit per sample causes more reduction than other quantization rates in BER as it gives BER equal to $9 * 10^{-4}$.

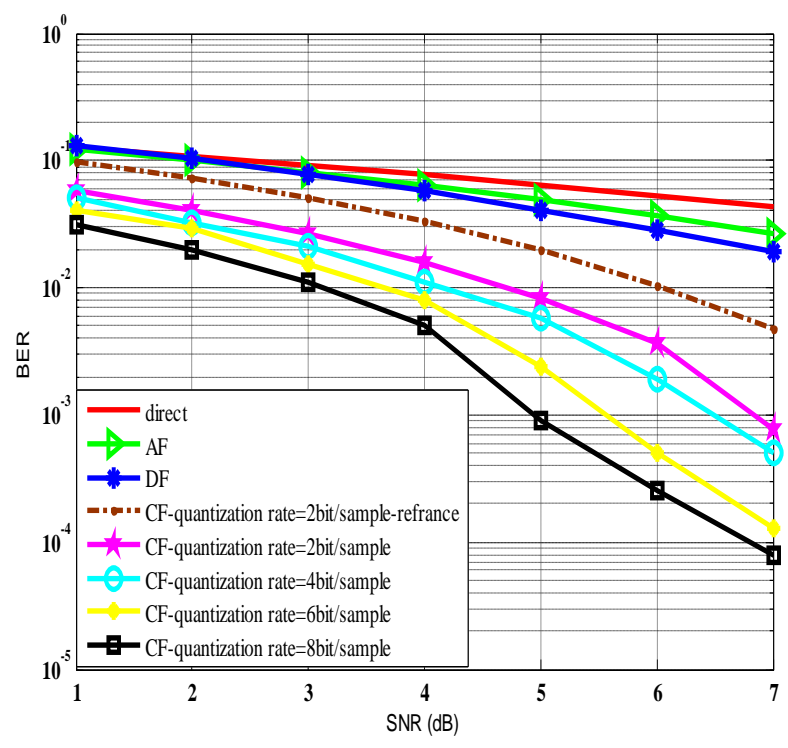

Fig.8: performances comparison for $\mathrm{AF}, \mathrm{DF}$ and $\mathrm{CF}$ with BPSK modulation scheme

\section{CONCLUSION}

A new implementation of $\mathrm{CF}$ for CCRN was presented. Based on joint (source-channel) coding and modulation, the proposed design allowed for cooperation with limited relay complexity. Proposed design that was used in this paper focused on providing good performance in the cases where neither DF nor AF fully had good performance due to low signal-to-noise ratios. Simulation results confirmed that remarkable gains could be achieved by using CF system over previously existing cooperation protocols. Multi-antennas multi-hopping techniques with decode and forward cooperative relays were introduced at BPSK modulation schemes in CCRN. It was obvious that using multi-hopping multi-antennas system improved the performance of the system than single antenna multihop system but if this number of hops increases over specified number, the interference will increase. To avoid this interference, the system will be more complex.

\section{REFERENCES}

[1] Thomas M. Cover and Abbas El Gamal, "Capacity Theorems for the Relay Channel", IEEE Transactions on Information Theory, pp. 572-584, 1979.

[2] R.U. Nabar, H. Bolcskei and F.W. Kneubuhler, "Fading Relay Channels: Performance Limits and Space-Time Signal Design", IEEE Journal on Selected Areas in Communication, vol. 22, no. 6, Aug. 2004.

[3] Blasco-Serrano, R. Thobaben, and M. Skoglund, "Bandwidth Efficient Compress-and-Forward Relaying Based on Joint Source Channel Coding", IEEE International Conf. on Communications, 2011. 
[4] Wyner and J. Ziv, "The Rate-Distortion Function for Source Coding with Side Information at The Decoder", IEEE Trans. Inf. Theory, vol. IT-22, no. 1, pp. 1-10, Jan. 1976.

[5] Host-Madsen and J. Zhang, "Capacity Bounds and Power Allocation for Wireless Relay Channels" ,IEEE Trans. Inf. Theory, vol. 51, no. 6, pp. 2020-2040, Jun. 2005.

[6] Nada Ebaid, Mona Shokair and Sami A. El Dolil, "Performance of Multihop Relaying System in Cognitive Radio Networks", International Journal of Computer Applications, vol. 81, no. 3, Nov. 2013.

[7] Nada Ebaid, Mona Shokair and Sami A. El Dolil, "Performance of Multi-Antennas Multi-hopping Relay in Cognitive Radio Network", Global Journal of Engineering, Design\& Technology ,vol.4, no. 3, pp1-4, August, 2014.

[8] O. Simeone, I. Stanojev, S. Savazzi, Y. Bar-Ness, U. Spagnolini and R. Pickholtz, "Spectrum Leasing to Cooperating Secondary Ad Hoc Networks", IEEE JSAC, vol. 26, no. 1,pp. $203-213,2008$.

[9] S. Hua, H. Liu, M. Wu and S. Panwar, "Exploiting MIMO Antennas in Cooperative Cognitive Radio Networks", INFOCOM, Proceedings IEEE, pp. 2714-2722, 2011.

[10] R. Blasco-Serrano, R. Thobaben, and M. Skoglund, "Compress and Forward Relaying Based on SymbolWise Joint Source-channel coding", IEEE International Conf. on Communications, June 2010.
[11] A. Dey, P. Kumar and K.Gupta, "on the Performance Analysis of Multi-Antenna Relaying System Over Rayleigh Fading Channel", ACEEE Int. J. on Control System and Instrumentation, vol. 02, no. 01, Feb. 2011.

[12] Conne, MinChulJu, Zhihang Yi, H.Song, and Il-Min Kim, "SER Analysis and PDF Derivation for Multi-Hop Amplify-and-Forward Relay Systems", IEEE Transaction on Wireless Communication, vol. 58, no. 8 , Aug. 2010.

[13] J. Jiang, John S. Thompson, Peter M. Grant, and Norbert Goertz, "Practical Compress-and-Forward Cooperation for The Classical Relay Network", 17th European Signal Processing Conference (EUSIPCO 2009), August 2009.

[14] R. M. Gray and D. Neuhoff, "Quantization" IEEE Transactions on Information Theory, vol. 44, no 6, pp. 2325-2383 Oct.1998.

[15] A. M. Benaya, Mona Shokair, El-Sayed El-Rabaie, and M. F. El-Kordy, "Relay-Based Throughput Maximization in Multiple Antennas Cognitive Radio Networks," 31th National Radio Science Conference (NRSC), pp. 116-123, Cairo, Egypt, April 2014

[16] A. M. Benaya, Mona Shokair, El-Sayed El-Rabaie, and M. F. El-Kordy, "Optimal Power Allocation for sensingBased Spectrum Sharing in MIMO Cognitive Relay Networks" ,Wireless Personal Communications, Springer, Feb. 2015.

[17] Hefdhallah Sakran, Mona Shokair, El-Sayed El-Rabaie, Omar Nasr and Atef Abou El-Azm, "Proposed Relay Selection Scheme for Physical Layer Security in Cognitive Radio Networks”, IET Communications, 2012. 